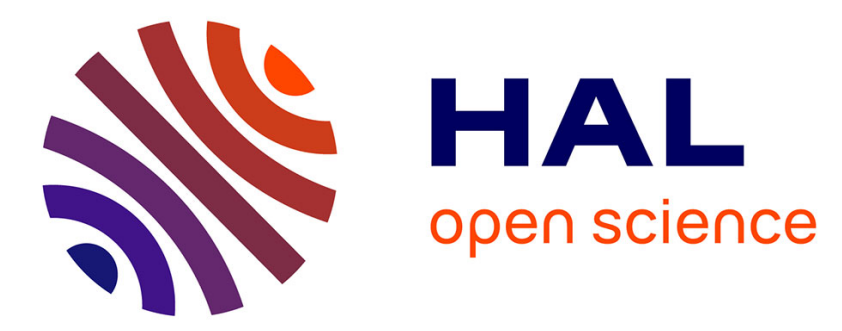

\title{
Inhibition of eIF5A hypusination pathway as a new pharmacological target for stroke therapy
}

Miled Bourourou, Elsa Gouix, Nicolas Melis, Jonas Friard, Catherine Heurteaux, Michel Tauc, Nicolas Blondeau

\section{- To cite this version:}

Miled Bourourou, Elsa Gouix, Nicolas Melis, Jonas Friard, Catherine Heurteaux, et al.. Inhibition of eIF5A hypusination pathway as a new pharmacological target for stroke therapy. Journal of Cerebral Blood Flow and Metabolism, 2020, pp.0271678X2092888. 10.1177/0271678X20928882 . hal-02899981

\section{HAL Id: hal-02899981 \\ https://hal.science/hal-02899981}

Submitted on 5 Nov 2020

HAL is a multi-disciplinary open access archive for the deposit and dissemination of scientific research documents, whether they are published or not. The documents may come from teaching and research institutions in France or abroad, or from public or private research centers.
L'archive ouverte pluridisciplinaire HAL, est destinée au dépôt et à la diffusion de documents scientifiques de niveau recherche, publiés ou non, émanant des établissements d'enseignement et de recherche français ou étrangers, des laboratoires publics ou privés.

\section{(ㄷ)(1) $\$$}

Distributed under a Creative Commons Attribution - NonCommerciall 4.0 International 


\title{
Inhibition of elF5A hypusination pathway as a new pharmacological target for stroke therapy
}

Journal of Cerebral Blood Flow \& Metabolism $0(0)$ I-II

(C) The Author(s) 2020 Article reuse guidelines: sagepub.com/journals-permissions DOI: $10.1177 / 0271678 \times 20928882$ journals.sagepub.com/home/jcbfm

@SAGE

\author{
Miled Bourourou I,*, Elsa Gouix ${ }^{1, *}$, Nicolas Melis², \\ Jonas Friard ${ }^{2}$ (D), Catherine Heurteaux', Michel Tauc ${ }^{2, \#} \mathbb{D}$ and \\ Nicolas Blondeau',\# (D)
}

\begin{abstract}
In eukaryotes, the polyamine pathway generates spermidine that activates the hypusination of the translation factor eukaryotic initiation factor 5A (elF5A). Hypusinated-elF5A modulates translation, elongation, termination and mitochondrial function. Evidence in model organisms like drosophila suggests that targeting polyamines synthesis might be of interest against ischemia. However, the potential of targeting elF5A hypusination in stroke, the major therapeutic challenge specific to ischemia, is currently unknown. Using in vitro models of ischemic-related stress, we documented that GC7, a specific inhibitor of a key enzyme in the elF5A activation pathway, affords neuronal protection. We identified the preservation of mitochondrial function and thereby the prevention of toxic ROS generation as major processes of GC7 protection. To represent a thoughtful opportunity of clinical translation, we explored whether GC7 administration reduces the infarct volume and functional deficits in an in vivo transient focal cerebral ischemia (tFCl) model in mice. A single GC7 pre- or post-treatment significantly reduces the infarct volume post-stroke. Moreover, GC7-post-treatment significantly improves mouse performance in the rotarod and Morris water-maze, highlighting beneficial effects on motor and cognitive post-stroke deficits. Our results identify the targeting of the polyamine-elF5A-hypusine axis as a new therapeutic opportunity and new paradigm of research in stroke and ischemic diseases.
\end{abstract}

\section{Keywords}

Brain ischemia, polyamine, elF5A, mitochondria, oxidative stress neuroprotection

Received II March 2020; Revised 24 April 2020; Accepted 26 April 2020

\section{Introduction}

Stroke is undoubtedly one of the worst devastating diseases worldwide with an enormous economic and societal burden. ${ }^{1}$ Although tremendous progress has been made in understanding the complex spatial and temporal mechanisms of major drivers of neuronal death in ischemic stroke - primarily excitotoxicity and oxidative stress - neuroprotective drug therapy targeting these and other mechanisms has been a dismal failure in clinical trials. ${ }^{2}$ While acknowledging several undesirable issues inherent in experimental stroke models and clinical trial designs, learning from efforts to save other organs from ischemic death can provide novel insight and therapeutic targets if applicable to the brain and ischemic stroke. ${ }^{3}$ For instance, in many experimental conditioning setups, ischemic tolerance has been achieved in multiple organs including the heart, kidney and brain and their respective cell types $^{4,5}$ and highlighted new putative targets.

In kidney ischemic injury, we recently reported the therapeutic potential of eukaryotic initiation factor

\footnotetext{
'CNRS, IPMC, Université Côte d'Azur, Sophia Antipolis, France

${ }^{2}$ CNRS, LP2M, Université Côte d'Azur, Nice, France

*These authors contributed equally to this work.

\#These authors share last author position.

Corresponding author:

Nicolas Blondeau, Institut de pharmacologie moléculaire et Cellulaire, CNRS, IPMC, 660 route des Lucioles, Sophia Antipolis, Valbonne 06560 , France.

Email: blondeau@ipmc.cnrs.fr
} 
5A (eIF5A) inhibition to protect against transient unilateral renal artery occlusion in rats and against anoxia in immortalized mouse renal proximal cells. ${ }^{6}$ Originally described for its role in initiating translation, hypusinated eIF5A not only promotes general translation elongation and termination, ${ }^{7,8}$ but also modulates mitochondrial function and oxygen consumption rates. ${ }^{9}$ eIF5A activation by hypusination is achieved by post-translational transfer and hydroxylation of the 4-amino butyl moiety from spermidine to a specific lysine residue ${ }^{10}$ by two key enzymes, known as deoxyhypusine synthase (DHS) and deoxyhypusine hydroxylase (DOHH). ${ }^{11}$ eIF-5A is the only known cellular substrate for DHS and the spermidine analog N1guanyl-1,7-diaminoheptane (GC7), a diaminoheptane derivative known as the most efficient inhibitor of DHS proved useful in protecting again renal ischemia in vivo and in vitro. Therefore, we investigated whether GC7 and underpinning DHS inhibition may lead to novel therapeutic opportunity in ischemic stroke.

\section{Material and methods}

\section{In vitro models of neuronal death}

Primary neuronal cultures containing less than 5\% glia were prepared from embryonic day 13.5 C57BL/12 mouse embryos. Dissociated cortical neurons were diluted in Neurobasal medium (Gibco) supplemented with $2 \% \mathrm{~B}-27,1 \%$ glutamax, $50 \mathrm{U} / \mathrm{mL}$ penicillin and $50 \mu \mathrm{g} / \mathrm{mL}$ streptomycin and seeded in 24-well plates. Addition of 5-fluoro-2'-deoxyuridin $(2 \mu \mathrm{M})$ was added at DIV1 to limit glia proliferation. The study by Melis et al. ${ }^{6}$ previously showed that $30 \mu \mathrm{M}$ was the lowest dose of GC7, which provided a maximum effect on anoxia-induced renal cell death. Therefore, neuronal cultures were treated with $30 \mu \mathrm{M}$ GC7 between 13 and 14 days in vitro (AtlanChim Pharma) for $12 \mathrm{~h}$ before the toxic insult.

Oxygen and glucose deprivation (OGD). To model ischemia in vitro, neuronal cultures were exposed to transient OGD for $60 \mathrm{~min}$ (lethal) to $120 \mathrm{~min}$ (supra-lethal). ${ }^{12,13}$ OGD was performed by placing the neuronal cultures in glucose-free medium $(116 \mathrm{mmol} / \mathrm{L} \mathrm{NaCl}, 5.4 \mathrm{mmol} / \mathrm{L}$ $\mathrm{KCl}, \quad 0.8 \mathrm{mmol} / \mathrm{L} \quad \mathrm{MgSO}_{4}, \quad 1 \mathrm{mmol} / \mathrm{L} \quad \mathrm{NaH}_{2} \mathrm{PO}_{4}$, $26.2 \mathrm{mmol} / \mathrm{L} \mathrm{NaHCO} 3,0.01 \mathrm{mmol} / \mathrm{L}$ glycine, $1.8 \mathrm{mmol} /$ $\mathrm{L} \mathrm{CaCl} 2 \mathrm{pH}$ 7.4) balanced with $1.2 \% \mathrm{O}_{2}\left(\mathrm{~N}_{2}\right.$ replacing $\left.\mathrm{O}_{2}\right)$ for 60 or $120 \mathrm{~min}\left(37^{\circ} \mathrm{C}, 5 \% \mathrm{CO}_{2}\right)$. OGD was stopped by returning to normoxic conditions in neurobasal medium.

NMDA, $\mathrm{H}_{2} \mathrm{O}_{2}$ and $\mathrm{KCl}$ toxicity. Stroke-related in vitro neuronal injuries were induced by either excitotoxicity with the glutamate agonist NMDA $(100 \mu \mathrm{M})$ for 30 or
60 min or superoxide production/oxidative stress by application of $300 \mu \mathrm{M} \quad \mathrm{H}_{2} \mathrm{O}_{2}$ for $30 \mathrm{~min}^{14}$ Neurotoxicity was also induced by chemical depolarization with an exposure to $50 \mu \mathrm{M} \mathrm{KCL}$ for $30 \mathrm{~min}^{15}$ Cultures were washed and returned to standard neurobasal medium. Neuronal death was estimated $24 \mathrm{~h}$ after the onset of the insult.

Assessment of neuronal death. Death of cultured neurons was quantified by trypan blue staining $24 \mathrm{~h}$ after exposure with an automatic counter (Life technologies) or using PI/Hoechst staining. PI-positive neurons were quantified with ImageJ software and expressed as a ratio of PI+ neurons/Hoechst + nuclei.

\section{Mitochondrial imaging}

To monitor the mitochondrial membrane potential $\left(\Delta \Psi_{\mathrm{m}}\right)$, vehicle or GC7-treated neurons seeded on fluorodishes were loaded with the cationic lipophilic dye tetramethylrhodamine ethyl ester (TMRE, Life Technologies) at $25 \mathrm{nM}$ for $20 \mathrm{~min}$. After washout of the dye and application of vehicle or NMDA $(100 \mu \mathrm{M})$, time-lapse imaging with a laser confocal LSM780 microscope (Carl Zeiss) was started with 5-min intervals for $60 \mathrm{~min}$. The mitochondrial superoxide production was measured using MitoSOX ${ }^{\mathrm{TM}}$ Red (Life technologies). Vehicle and GC7-treated neuronal cultures submitted or not to $60 \mathrm{~min}$ OGD were loaded with $3 \mu \mathrm{M}$ MitoSOX ${ }^{\mathrm{TM}}$ Red and $2 \mu \mathrm{g} / \mathrm{mL}$ Hoechst (Sigma Aldrich) $24 \mathrm{~h}$ after the onset of the insult. Acquisitions at $488 \mathrm{~nm}$ and $568 \mathrm{~nm}$ wavelengths were performed using videomicroscope (Carl Zeiss). TMRE and MitoSOX ${ }^{\mathrm{TM}}$ fluorescence intensity in individual neuron were analyzed with an image analysis software ( $n=12$ neurons and 3 separate neuronal cultures). Briefly, the area in each confocal micrograph occupied by each neuron was defined using the ImageJ 'threshold' function based on Hoechst staining, and the average fluorescence intensity derived from the indicator dyes (TMRM or MitoSOX Red) was measured within this area.

\section{Calcium imaging}

Intracellular calcium concentration $\left[\mathrm{Ca}^{2+}\right]_{\mathrm{i}}$ was determined by monitoring the fluorescence intensity of a calcium indicator Fura-2 AM (Molecular probe). Acquisition was performed after vehicle and GC7 treatment, and $24 \mathrm{~h}$ after an OGD challenge of neurons seeded at a density of $1,400,000$ cells/35-mm fluorodish. Following a 45-min dye loading with fluorescent probe Fura2-AM $\left(10 \mu \mathrm{M}\right.$, Life technologies) at $37^{\circ} \mathrm{C}$ in an atmosphere of $95 \%$ air $/ 5 \% \mathrm{CO}_{2}$, media was replaced by calcium imaging media containing (in $\mathrm{mM}$ ) 116 
$\mathrm{NaCl}$, 5.6 KCl, $1.2 \mathrm{MgCl}_{2}, 2 \mathrm{CaCl}_{2}, 20$ HEPES, $5 \mathrm{NaHCO}_{3}, 1 \mathrm{NaH}_{2} \mathrm{PO}_{4}$. Imaging was performed on fluorodishes mounted on an inverted fluorescent microscope (Zeiss) using a plan fluor 20X0.75 oil/water immersion fluorescent objective. For each condition, at least 50 neurons were recorded from three independent assays. The ratiometric imaging of Fura-2 fluorescence intensity reflecting the $\left[\mathrm{Ca}^{2+}\right]_{i}$ was obtained using probe excitation with alternative wavelengths of 340 and $380 \mathrm{~nm}$ and expressed as percentage of the respective control condition.

\section{Measurement of cellular ATP content}

Intracellular ATP level was measured using a luciferin/ luciferase-based assay (ATPlite, PerkinElmer) according to the manufacturer's instructions. Briefly, after vehicle and GC7 treatment, and $24 \mathrm{~h}$ after an OGD challenge, cortical neurons were gently swirled in lysis buffer before being incubated for $5 \mathrm{~min}$ with the substrate buffer. The emitted light was then detected with a Luminoskan $^{\mathrm{TM}}$ Ascent from Thermo. Total ATP was normalized against total protein determined by the BCA method (Thermo Fisher Scientific) to account for any difference in cell density and expressed as percentage of the respective control condition.

\section{Electrophysiology}

Membrane potentials were measured on cortical neurons. Cells were incubated during $12 \mathrm{~h}$ in the presence of vehicle (neurobasal) or GC7 $(30 \mu \mathrm{M})$, with or without $\mathrm{K}_{\mathrm{ATP}}$ channel blocker glibenclamide $(10 \mu \mathrm{M})$, the mitochondrial $\mathrm{K}_{\mathrm{ATP}}$ blocker 5-hydroxydecaoate $(5-\mathrm{HD}, 10 \mu \mathrm{M})$, apamin $(100 \mathrm{nM})$, or charybdotoxin $(100 \mathrm{nM})$. After the incubation period, cells were patched and membrane potentials were immediately measured using the whole cell patch clamp configuration (Hamill et al., 1981). ${ }^{16}$ Each membrane potential was evaluated by using a RK 400 patch clamp amplifier (Axon Instrument), low-pass filtered at $3 \mathrm{kHz}$ and digitized at $10 \mathrm{kHz}$ using a 12-bit analog-to digital converter digidata (1322 series, Axon Instrument, USA). Patch clamp pipettes were pulled using vertical puller (PC-10, Narishige) from borosilicate glass capillaries and had a resistance of 3-5 $\mathrm{M} \Omega$. The pipette solution contained (in mM) $155 \mathrm{KCl}, 3 \mathrm{MgCl}_{2}, 2.5 \mathrm{Na}_{2}$-ATP, 5 EGTA, 2.1 $\mathrm{CaCl}_{2}$, and 10 HEPES adjusted to $\mathrm{pH} 7.2$ with $\mathrm{KOH}$. All experiments were performed at room temperature $\left(21-22^{\circ} \mathrm{C}\right)$. Stimulation protocols and data acquisition were carried out using a microcomputer (Dell Pentium) which used a commercial software and hardware (pClamp 8.2).

\section{In vivo model of ischemic stroke}

Animals. Male eight-week-old C57BL/6J mice (Janvier France Breeding) were housed in a temperature- and humidity-controlled animal facility with a 12-h lightdark cycle. Food and water were available ad libitum. All efforts were made to minimize animal suffering and the number of animals used. All studies involving animal followed the guidelines outlined in European Community Directive 86/609/EEC, were approved by the Institutional Animal Care and Use Committee (CIEPAL-Azur, approval $\mathrm{n}^{\circ}$ 586-1560112863) and were completed in compliance with the ARRIVE guidelines for how to report animal experiments.

Model of transient focal cerebral ischemia. tFCI was induced in adult male mice (8-10-weeks-old, 25-30 g) by intraluminal occlusion of the right middle cerebral artery for 30 or $60 \mathrm{~min}$ with a 6-0 coated filament (Doccol, USA) as previously described. ${ }^{17}$ Experimental procedures were performed following criteria derived from Stroke Therapy Academic Industry Roundtable group guidelines for preclinical evaluation of stroke therapeutics, starting by treatments and all outcome assessments that were performed by investigators blinded to experimental group assignments. ${ }^{18}$ The regional cerebral blood flow was monitored by laser-Doppler flowmetry (Perimed, France) to standardize tFCI severity and confirm restoration of blood flow during reperfusion. Animals that did not show both criteria, CBF reduction of at least $85 \%$ of baseline level and reperfusion within $30 \mathrm{~min}$ after the filament withdrawal were excluded from the study.

Treatment with the eIFA5 hypusination inhibitor GC7. Animals were randomly assigned to vehicle or GC7 groups. Vehicle and GC7 were administrated intraperitoneally $(3 \mathrm{mg} / \mathrm{kg}){ }^{6}$ The pretreatment protocols consisted in three injections $48,24,2 \mathrm{~h}^{6}$ or a single injection $2 \mathrm{~h}$ before $60 \mathrm{~min}$ of tFCI. Post-treatment consisted in sequential injections $2 \mathrm{~h}, 2 \mathrm{~d}, 4 \mathrm{~d}, 7 \mathrm{~d}, 10 \mathrm{~d}, 14 \mathrm{~d}$ and $17 \mathrm{~d}$ after 30 min tFCI which is the recommended model for studying long-term functional outcomes in mice. ${ }^{19}$

Brain ischemic infarction. The infarct volume was assessed $24 \mathrm{~h}$ and 4 days after tFCI on 12 equally spaced coronal brain sections stained with cresyl violet. Tissue lesion was measured using a computer image analysis system and corrected for brain edema. Infarct volume (in $\mathrm{mm}^{3}$ ) was calculated by a linear integration of the corrected lesion areas. ${ }^{17,20}$

\section{Neurobehavioral performance}

Motor recovery. Sensorimotor deficits were evaluated by the rotarod test as previously described. ${ }^{17}$ Each mouse 
was subjected to four sessions: at $24 \mathrm{~h}$ before tFCI to determine the baseline (pre-operative score), and two, three and four days after tFCI. In each session, mice were trained on the rotarod for $5 \mathrm{~min}$ at a constant speed $(4 \mathrm{r} / \mathrm{min})$, after $30 \mathrm{~min}$ rest, they were then subjected to a single trial to measure their latency to fall from the rotating rod (diameter, $30 \mathrm{~mm}$ ) that was accelerated from 4 to $40 \mathrm{r} / \mathrm{min}$ over $5 \mathrm{~min}$, the trial being stopped after $10 \mathrm{~min}$.

Cognitive recovery. Long-term cognitive deficits were evaluated in the Morris water maze at 14-18days after $\mathrm{tFCI}$, in a 120 -cm-diameter, 60 -cm-high circular swimming pool filled to a depth of $32 \mathrm{~cm}$ with $21 \pm 2^{\circ} \mathrm{C}$ opaque water, as previously described. ${ }^{17}$ Learning deficits were evaluated measuring the time needed for the animal to locate the submerged platform (escape latency) from 14 to 17 days after tFCI. Spatial memory deficits were evaluated at D18 after tFCI by measuring the time spent in the target quadrant when the platform was removed. Swim pattern of the probe test at D18 was recorded by ANY-maze software (Version 5.6; Stoelting Co., Wood Dale, Il).

Statistical analyses. Statistical analysis was performed using GraphPad Prism 6.0 (GraphPad Software, La Jolla, CA, USA). Results are presented as means \pm 95\% confidence intervals (CI) for the in vitro experiments and as mean \pm SD for the in vivo experiments. Unpaired $t$ test and Mann-Whitney test were performed to assess the difference between groups as appropriate. Two-way ANOVA was performed when two groups (first factor) were compared over the time (second factor). This test was used to compare the GC7 effect on motor recovery and cognitive recovery. Statistically significant value was set to $p<0.05$, ${ }^{*} p<0.05,{ }^{* *} p<0.01,{ }^{* * *} p<0.001$.

\section{Results}

\section{GC7 decreases neuronal death induced in vitro by oxygen-glucose deprivation and ischemic-related stress}

A potential anti-ischemic effect of GC7 was first evaluated in vitro, by using primary cultures of cortical neurons submitted to oxygen-glucose deprivation (OGD). GC7 treatment $(30 \mu \mathrm{M})$ provided significant neuroprotection against 60-min OGD and even against a more severe 120-min OGD insult (Figure 1(a)). GC7 protection against OGD-induced necrosis was confirmed based on use of the cell death marker propidium iodide (PI) measured during $24 \mathrm{~h}$ following reperfusion. Reductions by factors of 4.5, 1.9 and 1.8 in the
PI-positive neurons were observed immediately, $6 \mathrm{~h}$ and $24 \mathrm{~h}$ after OGD (Figure 1(b)), suggesting an inhibition of multiple steps in the neurodegenerative cascade by GC7-based inhibition of eIF5A hypusination. OGD induces energy failure, thereby disrupting transmembrane ion gradients and mitochondrial function. Specifically, extracellular glutamate elevations causes over-activation of N-methyl-D-aspartate (NMDA) glutamate receptors, promoting excessive membrane depolarization, calcium and sodium entry in the cell and calcium release from the mitochondria, and reactive oxygen toxicity. ${ }^{5}$ We therefore investigated whether GC7 could protect primary neuronal culture against NMDA glutamate receptor- and hydrogen peroxideinduced neurotoxicity. GC7 treatment fully abolished neuronal cell death induced by a 30-min exposure to $100 \mu \mathrm{M}$ NMDA and reduced neuronal death induced by a 30 -min exposure to $300 \mu \mathrm{M} \mathrm{H}_{2} \mathrm{O}_{2}$ from $33 \%$ to $15 \%$, measured $24 \mathrm{~h}$ after reperfusion (Figure $1(\mathrm{c})$ ). We also evaluated GC7 efficiency against neuronal death induced by KCL depolarization (Figure 1(c)), which causes voltage-gated L-type $\mathrm{Ca}^{2+}$ channels to open, triggering an overwhelming influx of $\mathrm{Ca}^{2+}$ entry into neurons. GC7 treatment failed to protect against $50 \mu \mathrm{M} \mathrm{KCl}$ challenge. This toxic pathway differs from the mitochondrial collapse induced by OGD and NMDA receptor overactivation. ${ }^{21,22}$ Taken together, the data point to mitochondrial protection as a pivotal step in protection by GC7.

\section{GC7 protects mitochondria from membrane potential failure}

Since mitochondria represents an appealing target for the development of new neuroprotective pharmacological interventions, ${ }^{23,24}$ we then focus on GC7-effect on mitochondrial function, using TMRE, a voltagesensitive marker that preferentially accumulates into the mitochondria due to the negative $\Delta \Psi \mathrm{m}^{25}$ Strikingly, the decrease in $\Delta \Psi \mathrm{m}$ induced by $30 \mathrm{~min}$ of NMDA challenge was significantly prevented by GC7 (Figure 1(d)). To elucidate the temporal window of protection, we evaluated if GC7 preserved $\Delta \Psi \mathrm{m}$ even when the NMDA challenge was administered late after GC7 elimination from the medium (48 and $72 \mathrm{~h}$ ). The loss of about $80 \%$ of TMRE fluorescence was still abolished $48 \mathrm{~h}$ after GC7 treatment, whereas $72 \mathrm{~h}$ after GC7 treatment no further protection of the $\Delta \Psi \mathrm{m}$ against NMDA depolarization was provided (Figure 1(d)). This identifies steady and long-lasting beneficial effects of GC7 on mitochondria integrity in GC7-treated neuronal cultures. These may not only prevent neuronal death during ischemia, but may also facilitate maintenance and recovery of neuronal function that are ATP dependent. 


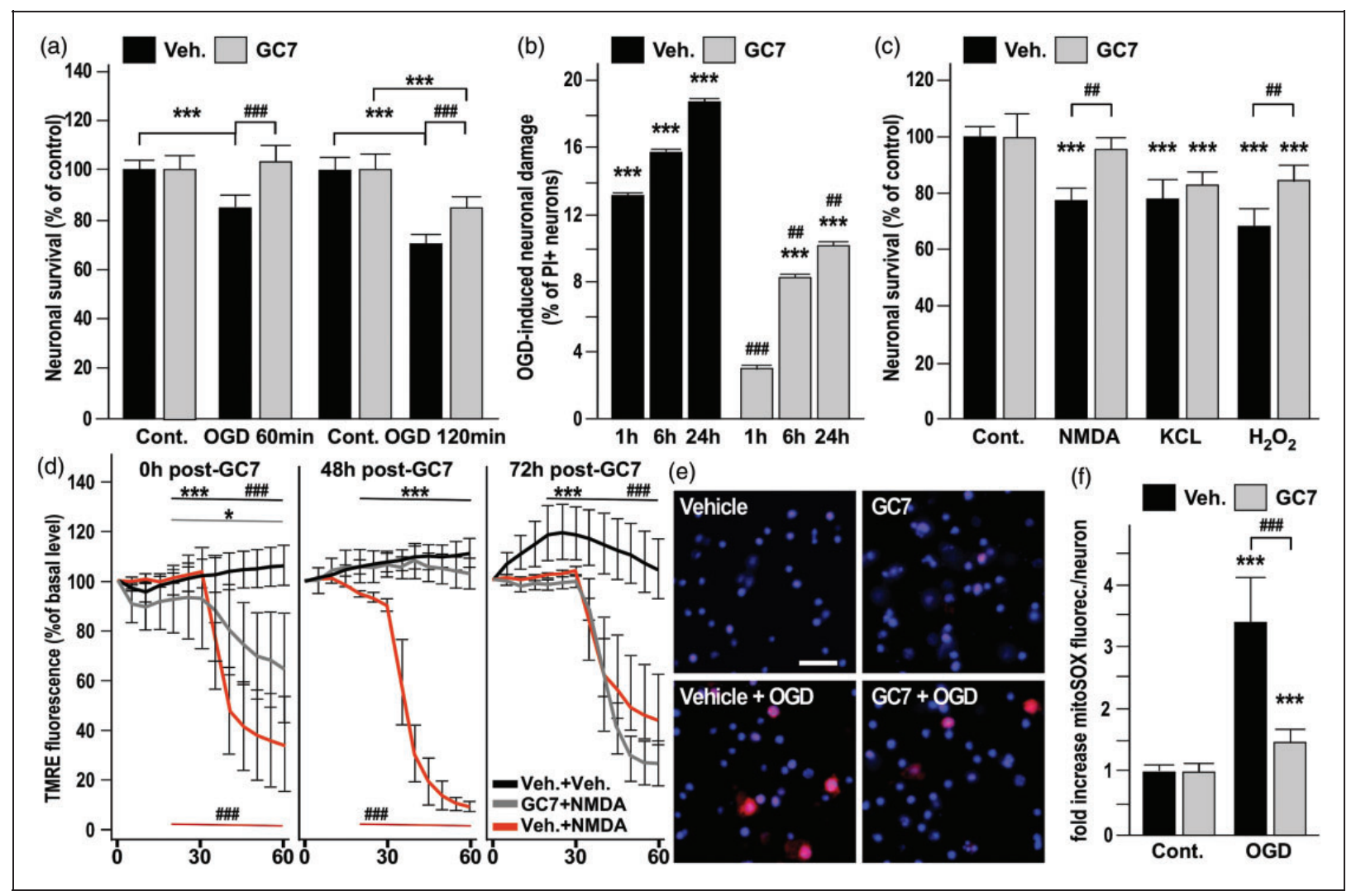

Figure I. GC7 decreases neuronal death induced in vitro by ischemic-related stress by protecting mitochondria from membrane potential failure and ROS generation. (a-b) Cortical neurons exposed to GC7 were more resistant to 60 and I20 min OGD (a) and display a reduced PI-uptake measured during $24 \mathrm{~h}$ following $120 \mathrm{~min}$ OGD (b). (c) Protective effect of GC7 against 30-min exposure to $100 \mu \mathrm{M}$ NMDA and to $300 \mu \mathrm{M} \mathrm{H}_{2} \mathrm{O}_{2}$, but not to $50 \mu \mathrm{M} \mathrm{KCL}$. (d) TMRE fluorescence is preserved in the GC7-treated neurons indicating a protective effect in preventing the loss of $\triangle \Psi \mathrm{m}$ induced by NMDA, immediately after and $48 \mathrm{~h}$, but not $72 \mathrm{~h}$ after the GC7 pretreatment. ${ }^{*} p<0.05$ and $*^{* * *} p<0.001$ versus respective vehicle (Veh.) +NMDA-treated condition. \#\#p $<0.00$ I versus

GC7+NMDA-treated condition. (e, f) GC7 reduces OGD-induced mitochondrial superoxide generation in primary neurons stained with MitoSOX ${ }^{\mathrm{TM}}$, as shown by representative micrographs (e) and quantification of relative red fluorescence per neurons $24 \mathrm{~h}$ after OGD (f). $* * * p<0.001$ versus respective control basal condition. \#p<0.01, \#\#p<0.001 versus vehicle-treated condition.

\section{Preservation of mitochondria membrane potential and thereby of prevention of ROS generation are converging points for GC7 protective effect}

The loss of $\Delta \Psi \mathrm{m}$ is a hallmark of neuronal cell death linked to mitochondrial dysfunction causing excessive ROS production, elevated extra-mitochondrial $\left[\mathrm{Ca}^{2+}\right]$, and decreased $[\mathrm{ATP}]_{\mathrm{i} .}{ }^{26,27}$ The above mitochondrial results suggested that $\mathrm{GC} 7$ might also protect against these three prominent features occurring at the early stages of acute glutamate excitotoxicity and ischemic stroke (in both the necrotic and apoptotic components of stroke).

We therefore explored the potential of GC7 to reduce OGD-induced oxidative stress and superoxide generation in mitochondria, using the mitochondrial selective dye MitoSOX Red. ${ }^{28}$ Remarkably, the increase in superoxide levels observed after OGD was strongly prevented in neurons treated with GC7
(Figure 1(e) and (f)), confirming a comparable mechanism of protection by inhibiting ROS production in both renal ${ }^{6}$ and neuronal cells. In addition, mitochondria protection by GC7 prevented OGD-induced $\left[\mathrm{Ca}^{2+}\right]_{\mathrm{i}}$ accumulation (Figure 2(a)) and $[\mathrm{ATP}]_{\mathrm{i}}$ drop (Figure 2(b)). Since the correlated rise in $\left[\mathrm{Ca}^{2+}\right]_{\mathrm{i}}$ and drop in ATP are initiated by depolarization of the neuronal membrane in response to ischemia, our observation raise the possibility that GC7 displayed an unexpected beneficial action on membrane potential of neurons that prevents them from ischemic depolarization. Therefore, we further evaluated the effect of GC7 on the resting membrane potential. Whole-cell patch clamp recordings in cortical neurons indicated that GC7 induces membrane hyperpolarization (Figure 2(c)) that could be attributed to the opening of $\mathrm{K}^{+}$channels, an inherent protective mechanism constituting another exciting target for neuroprotection. ${ }^{29}$ Pharmacological inhibition of specific $\mathrm{K}^{+}$currents 


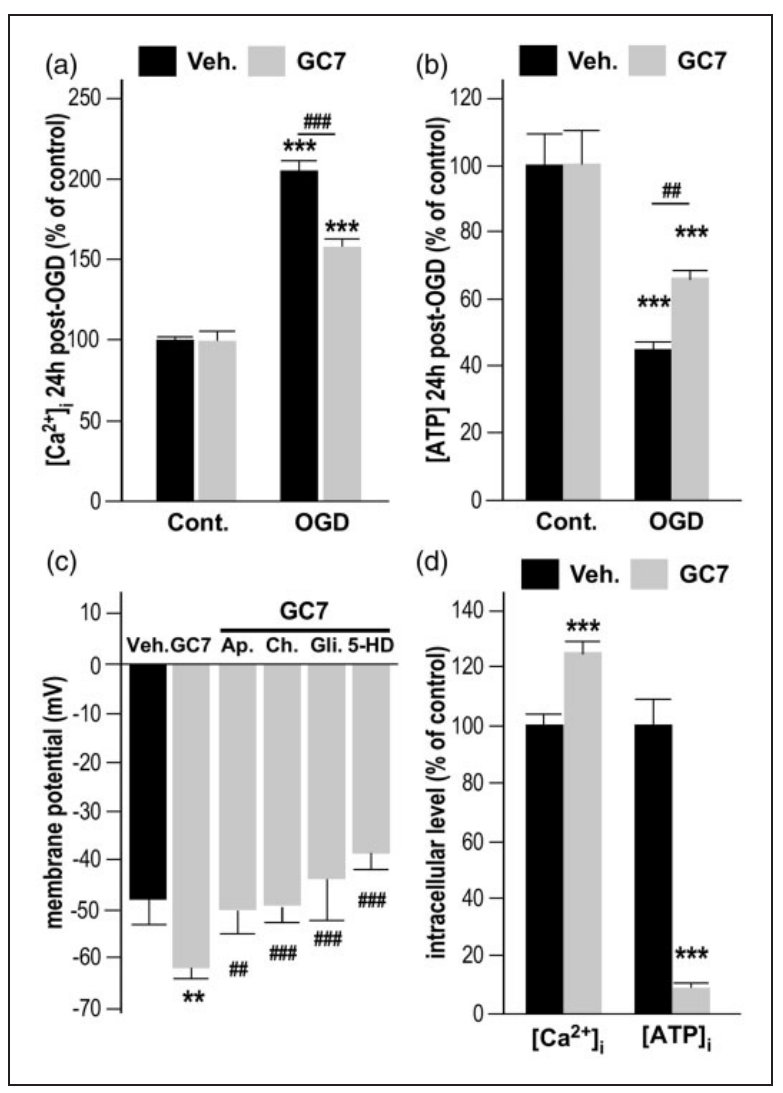

Figure 2. GC7 preserves the cellular energetic state and the membrane potential. (a) GC7 reduces $\left[\mathrm{Ca}^{2+}\right]_{\mathrm{i}}$ accumulation in cortical neurons exposed to OGD. (b) GC7 also reduces OGDinduced $[A T P]_{i}$ drop. (c) GC7 induces a membrane hyperpolarization that was fully blocked by the specific $\mathrm{Ca}^{2+}$-activated $\mathrm{K}+$ $(\mathrm{K}(\mathrm{Ca}))$ channel blocking toxins apamin $(100 \mathrm{nM})$ and charybdotoxin $(100 \mu \mathrm{M})$, the $\mathrm{K}_{\text {ATP }}$ channel blocker glibenclamide ( 10 $\mu M)$, and the mitochondrial $K_{\text {ATP }}$ channels blocker 5-hydroxydecanoate (5-HD, $10 \mu \mathrm{M})$. (d) When cultured in $\mathrm{Ca}^{2+}$-lacking medium, GC7-treated neurons display an elevated $\left[\mathrm{Ca}^{2+}\right]_{i}$ and lower ATP production, corroborating an adaptation of the mitochondrion to GC7 treatment. ${ }^{* * *} p<0.001$ versus respective control (cont.) condition. \#p<0.0I, \#\#p<0.00I versus vehicle-treated condition.

showed that GC7-induced hyperpolarization was fully blocked by the specific $\mathrm{Ca}^{2+}$-activated $\mathrm{K}^{+}(\mathrm{K}(\mathrm{Ca}))$ channel toxins apamin $(100 \mathrm{nM})$ and charybdotoxin $(100 \mathrm{nM})$, and the $\mathrm{K}_{\mathrm{ATP}}$ channel blocker glibenclamide $(10 \mu \mathrm{M})$, suggesting that GC7 did not target a specific $\mathrm{K}^{+}$channel at the membrane, but that the enhanced neuronal $\mathrm{K}^{+}$conductance at the membrane may occur in response to changes in intracellular concentrations of ATP or $\mathrm{Ca}^{2+}$. The $\mathrm{K}_{\text {ATP }}$ channels in the mitochondrial membrane are known to link the cellular energetic state to the membrane potential. ${ }^{30}$ Their specific blockade by 5-hydroxydecanoate $(5-\mathrm{HD}, 10 \mu \mathrm{M})$ also fully prevented GC7-induced hyperpolarization (Figure 2 (c)), pinpointing the mitochondrion as a converging

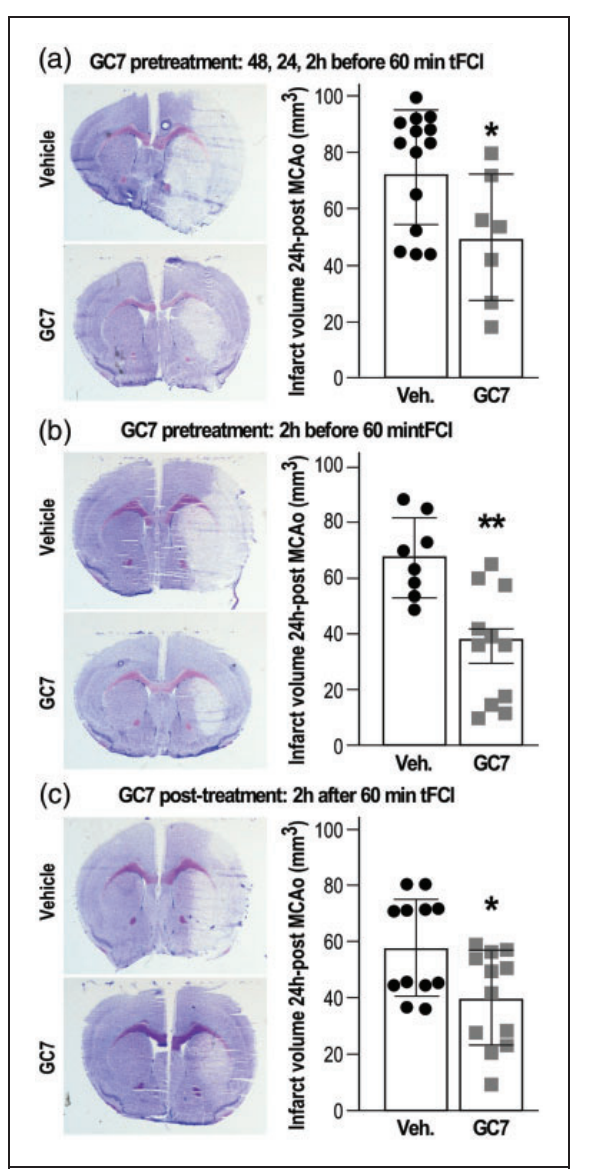

Figure 3. GC7 intraperitoneal injection reduces $\mathrm{tFCl}$-induced infarct volume. (a) Three injections of GC7 (3 mg/kg daily for three days) or (b) a single injection $2 \mathrm{~h}$ before $\mathrm{tFCl}$ or (c) a single post-treatment $2 \mathrm{~h}$ after $\mathrm{tFCl}$ significantly reduce $\mathrm{tFCl}$-induced lesion as illustrated by representative cresyl violet staining and quantitation of the infarct volume. $N=7-12 /$ group, $* p<0.05$, $* * p<0.01$ versus vehicle-injected mice.

point for GC7 protective effect. Indeed, the beneficial effect of GC7 strengthening the inherent protective action of increase $\mathrm{K}^{+}$conductance is probably a byproduct of GC7 effect on mitochondria related to lowered ATP production and increased $\left[\mathrm{Ca}^{2+}\right]_{i}$ in neurons cultured in $\mathrm{Ca}^{2+}$-deprived medium (Figure 2(d)).

\section{Both GC7 intraperitoneal pre- and post-treatment reduce brain lesion after stroke}

Thus far, GC7 targeting of the mitochondria successfully achieved experimental in vitro neuronal protection through reducing mitochondrial dysfunction and ROS generation, $\mathrm{Ca}^{2+}$ accumulation, and ATP reduction. Given its in vivo beneficial effect when injected $(3 \mathrm{mg} / \mathrm{kg}$ daily for three days, intraperitoneal (IP)) prior to 40 min transient unilateral renal artery ischemia, ${ }^{6}$ we hypothesized that an IP injection of GC7 could protect 


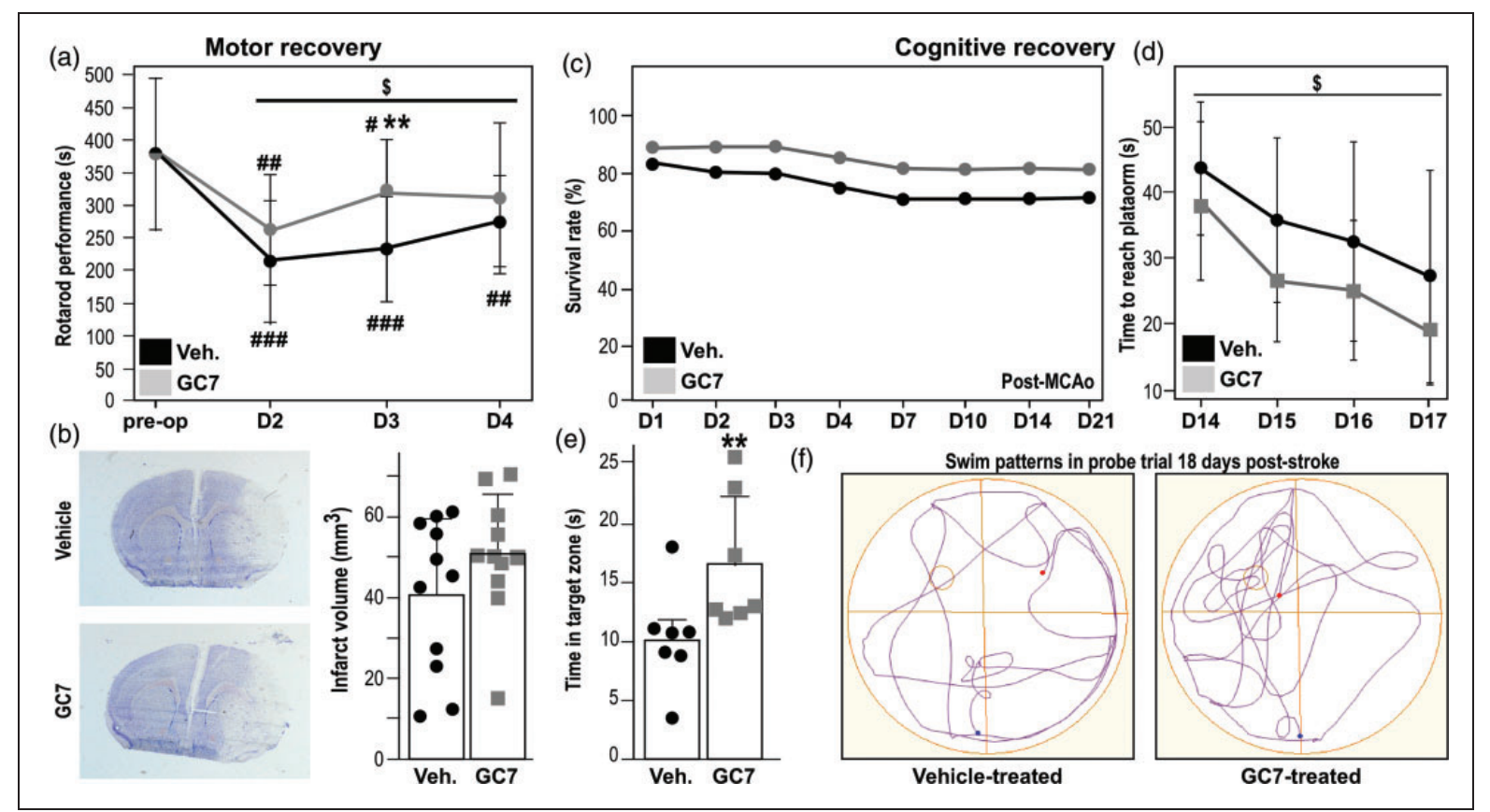

Figure 4. GC7 post-treatment reduces motor and cognitive impairment post-stroke. (a) The post-tFCl rotarod performance of GC7-treated mice is significantly improved during the four days of recovery (2W ANOVA: effect of group: $F=4.1 \mathrm{I}, \$ p=0.04594$ ). $n=$ I I per group at each time point) \#p<0.05; \#p<0.0 I; \#\# <0.00I versus pre-tFCl performance; ** $p<0.0$ I different from vehicletreated control. (b) Histological examination of brain sections after $30 \mathrm{~min} \mathrm{tFCl}$ did not relate motor dysfunctions improvement with any reduction of the infarct $(n=\mathrm{II}$ per group). (c) Long-term survival rate after $30 \mathrm{~min}$ of $\mathrm{tFCl}$ is similar between GC7 and vehicletreated groups ( $n=14$ in each group). (d) In the Morris Water-Maze test, during the learning period, GC7 post-treatment improves latency to find the hidden platform ( 2 W ANOVA: effect of group: $F=5.13, \$ p=0.0263$ ), and (e) during the probe trial, GC7 posttreatment increased the time spent by mice in the target quadrant (platform removed), ${ }^{* *} p<0.0 \mathrm{I}, \mathrm{N}=7$ in each group. (f)

Representative swim patterns 18 days post-stroke illustrate that GC7-treated mice display a better probe strategy.

the brain against acute ischemic stroke in a clinically relevant transient focal cerebral ischemia (tFCI) and reperfusion model. CG7 should share the same properties of polyamines in demonstrating some permeability across the blood-brain barrier ${ }^{31}$ and the strokeinduced alteration of the blood-brain barrier ${ }^{32}$ should further greatly enhance GC7 permeability. We found that not only a similar pretreatment protocol by three injections 48, 24, $2 \mathrm{~h}$ before tFCI (Figure 3(a)), but also a single injection $2 \mathrm{~h}$ before tFCI, reduced cerebral infarction size (Figure 3(b)). Moreover, a single IP injection of $\mathrm{GC} 7$ provided $2 \mathrm{~h}$ after reperfusion induced a similar brain protection (Figure 3(c)).

\section{GC7 post-treatment improves long-term functional recovery}

The successful translation of new therapeutic opportunities will depend on their efficacy on functional strokeinduced deficits. We therefore evaluated the effect of GC7 post-treatment in the 30-min tFCI model, which is recommended for studying long-term functional outcomes in mice. ${ }^{19}$ In this model, the temporary impairment of motor function (animals showed near baseline recovery by day 4 post-tFCI) correlates to an anatomical reorganization of undamaged brain areas and not to a decrease of infarct volume. ${ }^{19} \mathrm{GC} 7$ post-treatment (IP injection $2 \mathrm{~h}$ and two days post-tFCI) facilitated post-stroke recovery of motor functions assessed using rotarod (Figure 4(a)). As expected, the histological examination of brain sections four days after tFCI (Figure 4(b)) did not relate motor dysfunctions improvement with any reduction of the infarct. As this result indicated an overall better preservation of neuronal function by GC7, we further determined the effect of GC7 on cognitive deficits using the Morris water-maze test at two weeks after surgery. In the 30min tFCI, the low long-term mortality that is too low to be improved by any treatment, ${ }^{17}$ was not influenced by GC7 post-treatment (Figure 4(c)), while distinct beneficial effects could be discriminated on long-term impairment of spatial learning (Figure 4(d)) and memory (Figure 4(e)). Overall, GC7-treated mice exhibited improved searching behaviors traveling more distance in the target quadrant where the platform was previously located compared with the vehicletreated mice (Figure 4(f)). 


\section{Discussion}

In vitro primary neuronal culture treated by GC7, the best-known inhibitor of DHS exhibited reduced cell death associated with ischemic-related stress, such as OGD, NMDA excitotoxicity and oxidative stress. The GC7 anti-ischemic effect was also found against a gold standard in vivo model of stroke. Using different tFCI durations, we demonstrated that GC7 treatment improves both neuronal survival and spontaneous functional recovery after stroke. To the best of our knowledge, it is the first proof of concept that pharmacological interfering with the polyamine-eIF5Ahypusine axis with GC7 might have novel implications for therapeutic controlling of stroke consequences. Beyond this primarily goal, our study provides new mechanistic insights identifying mitochondria as the ultimate destination in GC7 anti-ischemic effects. This echoes a recent discovery intricately linking eIF5A and cellular metabolism and respiration. ${ }^{9}$ The fact that acute inhibition of eIF5A hypusination could dampen the Krebs cycle that highly needs oxygen for functioning while leaving glycolytic ATP synthesis operative $^{9}$ may be salutary in ischemic conditions. Consistent with these observations, by preventing the complete oxidation of glucose that produces large amount of ATP in the mitochondria compared to glycolysis, GC7 dampens the ATP production in neurons in physiological condition, while maintaining sufficient ATP to sustain transitory cell membrane integrity, functioning and survival under OGD. Moreover, it was recently suggested that, in hypoxic-ischemic brain injury, outcomes would decline as mitochondrial function becomes more dependent on oxygenation. ${ }^{33}$ Therefore, by modifying the mitochondria dependency for oxygen supply, which is normally critical for the survival of neurons, GC7 treatment may improve the metabolism at the cellular level at a lower oxygenation level, resulting in a more favorable outcome.

In ischemic stroke where oxygen and energy supply are temporary defective, glycolysis-derived lactate, a crucial energy source used by neurons and other cells types was recently recognized for dampening neuronal excitability, triggering neuroprotection and improving neuronal plasticity. ${ }^{34}$ Not only it is possible that GC7 also exerts a direct protective effect on astrocytes, but improving neuronal survival and function should help support the metabolic coupling between neurons and astrocytes and therefore the dynamic cooperation between these two cell types. Maintaining some neuronal activity would stimulate astrocytic lactate formation and release to support the metabolic/functional needs of adjacent neurons and oligodendrocytes. ${ }^{35}$

Another type of positive feedback loop between neutrons and astrocytes - and even other cell types - that may also boost the tissue viability by helping with the functionality of surrounding cells is the transfer of healthy mitochondria. ${ }^{36}$ Since the consensus that mitochondrial function reflects stroke disease states, and is readout of neurobehavioral recovery, transfer of healthy mitochondria to ischemic cells has become an appealing therapeutic approach for stroke that may account for the protective action of stem cell therapy. ${ }^{37}$ Therefore, GC7-induced preservation of functional mitochondria fosters a therapeutic pool of healthy mitochondria available for transfer to dampen secondary cell death processes, including ROS generation and inflammation. Overall, re-orienting cells metabolism to adapt to the ischemic situation, the inhibition of eIF5A hypusination may present a crucial advantage in the less-severely ischemic "penumbral" tissue that suffer more of the limited availability of oxygen than glucose to preserve the cell and mitochondria integrity.

While our new results on mitochondrial membrane potential, ROS generation and ATP production reveal the potential of GC7 to preserve mitochondrial function after stroke, another parameter that would have been interesting to measure is the post-stroke mitochondrial oxygen consumption rate (OCR) with and without GC7 treatment in both the parenchyma and the cerebral vessels. Indeed, the response or sensitivity to ischemia/reperfusion may be different depending on the cell type, especially in vessels. Moreover following experimental stroke, OCR increases in the ischemic middle cerebral arteries (MCAs) compared with contralateral and naïve MCAs. ${ }^{38}$ Showing that GC7 could correct such stroke-induced change in MCAs OCR would further support the concept that GC7 protection against ischemia may extend to the cerebro-vasculature by preserving mitochondrial bioenergetic function.

Interestingly, mitochondria is recognized as a common and crucial biochemical target of several FDA-approved drugs that have not been followed up clinically for stroke, while available and/or approved for other applications. ${ }^{39}$ Therefore, our study should lead to increasing interest in developing and testing new inhibitors of eIF5A and rapidly translate to patient care, considering the well-documented safety and tolerability of the compounds targeting the polyamine biosynthetic pathway. Indeed, some of them are extensively studied in cancer, ${ }^{40,41}$ including in phase I clinical trial for neuroblastoma, the most common cancer in children, ${ }^{42}$ non-small cell lung cancer ${ }^{43}$ and metastatic breast cancer. ${ }^{44}$ A striking example is DFMO, $\alpha$-difluoromethylornithine a pharmacological inhibitor of the ornithine decarboxylase, which was used for the treatment of African sleeping sickness ${ }^{45}$ and positively evaluated in phase III randomized trial against anaplastic gliomas ${ }^{46}$ and is also under investigation as a chemopreventive agent ${ }^{47}$ was shown to 
protect both cardiomyoblasts ${ }^{48}$ and neuronal cultures against ischemia. ${ }^{49}$ Likewise, targeting eIF5A is under investigation for treating several human cancers ${ }^{50}$ and the safety and tolerability of intravenous infusion of chemical inhibitor of eIF5A have already been evaluated in Phase I-II in patients with relapsed or refractory B-cell malignancies. ${ }^{51}$ Taking advantage of the clinical data on pharmacokinetics, toxicity, safety and tolerability (e.g. knowledge of the absence of dose limiting toxicities, DLTs) would serve as basis in shaping the direction of novel "drug repurposing/ repositioning" research study, in which a clinically approved drug is investigated for a novel application that is for the present application of its therapeutic potential in ischemic stroke.

Interestingly, the specific inhibitory effect of $\mathrm{GC} 7$ on eIF5A hypusination has been investigated in different organs, in both male and female, in mice ${ }^{52,53}$ and rats ${ }^{6}$ and these previous in vivo studies in mice showed evidence that GC7 does not induce noticeable side effects. Nevertheless, based on sex differences to stroke vulnerability $^{54}$ and, perhaps, to neuroprotective treatment as well, the effect of GC7 should be tested in both sexes for safety and tolerability. Despite GC7, the bestknown inhibitor of DHS displaying an IC50 of $17 \mathrm{nM},{ }^{55}$ was not used in human clinical trials; some studies with molecules exhibiting an inhibitory effect on DHS have been published and used in clinical trials. Semapimod also called CNI-1493 or AXD455 inhibits DHS with an IC50 around $2 \mu \mathrm{M} .{ }^{56}$ Semapimod that is well tolerated by patients ${ }^{57}$ was tested for Crohn's disease ${ }^{58,59}$ and for the consequence of Endoscopic Retrograde CholangioPancreatography. ${ }^{60}$ From these studies led in humans, it is tempting to reasonably hypothesize that acute targeting of DHS with a compound like GC7 presenting an higher affinity than those use in clinical trial should not lead to significant adverse effects. Moreover, the effectiveness of stroke reperfusion therapy remains limited because the majority of stroke patients lack timely access to intravenous thrombolysis or endovascular therapy, underscoring an unmet clinical need for adjunctive neuroprotective treatments. ${ }^{61}$ Delaying the need for oxygen, thereby "buying time" by extending the therapeutic window for reperfusion therapy, makes this an innovative and promising pharmacological agent for future clinical protocols related to ischemic challenges in combination with reperfusion therapy.

Thereby, our findings should contribute accelerating clinical trial development by proposing a new research opportunity circumventing crucial issues regarding the translation of preclinical developments to the bedside.

Finally, the novelty of eIF5a as a therapeutic target against brain ischemia and the potential of GC7 in stroke therapy advocate potential promise in screening for GC7-structurally-related eIF5A hypusinationinhibitors and targeting signaling pathways altered by GC7 as a potential therapeutic strategy in humans suffering from ischemic diseases.

\section{Funding}

The author(s) disclosed receipt of the following financial support for the research, authorship, and/or publication of this article: This work was supported by a grant DPM 20121125559 from the Fondation pour la recherche médicale (FRM), and a grant from the Société d'Accélération de Transfert de Technologie (ValorPACA/SATT Sud-Est) from France.

\section{Acknowledgements}

We are very grateful to Dr. Hamid Moha Ou Maati for his skillful technical support and advices with the electrophysiology, Dr. Edith Hamel and Dr. Joseph S. Tauskela for their critical reading of the manuscript.

\section{Declaration of conflicting interests}

The author(s) declared no potential conflicts of interest with respect to the research, authorship, and/or publication of this article.

\section{Authors' contributions}

$\mathrm{MB}, \mathrm{EG}, \mathrm{NM}, \mathrm{JF}$ and $\mathrm{NB}$ performed the experiments. MB, EG, MT and NB analyzed the data. $\mathrm{MB}, \mathrm{CH}, \mathrm{MT}$ and $\mathrm{NB}$ wrote the paper.

\section{ORCID iDs}

Jonas Friard (D) https://orcid.org/0000-0002-5260-8056

Michel Tauc (D) https://orcid.org/0000-0002-8383-9818

Nicolas Blondeau (D) https://orcid.org/0000-0001-5954-4094

\section{References}

1. Feigin VL, Norrving B and Mensah GA. Global burden of stroke. Circul Res 2017; 120: 439-448.

2. Lo EH, Dalkara T and Moskowitz MA. Mechanisms, challenges and opportunities in stroke. Nat Rev Neurosci 2003; 4: 399-415.

3. Moskowitz MA, Lo EH and Iadecola C. The science of stroke: mechanisms in search of treatments. Neuron 2010; 67: 181-198.

4. Gidday JM. Cerebral preconditioning and ischaemic tolerance. Nat Rev Neurosci 2006; 7: 437-448.

5. Iadecola $C$ and Anrather J. Stroke research at a crossroad: asking the brain for directions. Nat Neurosci 2011; 14: 1363-1368.

6. Melis N, Rubera I, Cougnon M, et al. Targeting eIF5A hypusination prevents anoxic cell death through mitochondrial silencing and improves kidney transplant outcome. J Am Soc Nephrol 2017; 28: 811-822. 
7. Saini P, Eyler DE, Green R, et al. Hypusine-containing protein eIF5A promotes translation elongation. Nature 2009; 459: 118-121.

8. Schuller AP, Wu CC, Dever TE, et al. eIF5A functions globally in translation elongation and termination. Mol Cell 2017; 66: 194-205.e5.

9. Puleston DJ, Buck MD, Klein Geltink RI, et al. Polyamines and eIF5A hypusination modulate mitochondrial respiration and macrophage activation. Cell Metab 2019; 30: 352-363.e8.

10. Cano VS, Jeon GA, Johansson HE, et al. Mutational analyses of human eIF5A-1 - identification of amino acid residues critical for eIF5A activity and hypusine modification. FEBS J 2008; 275: 44-58.

11. Park MH, Liberato DJ, Yergey AL, et al. The biosynthesis of hypusine ( $\mathrm{N}$ epsilon-(4-amino-2-hydroxybutyl) lysine). Alignment of the butylamine segment and source of the secondary amino nitrogen. J Biol Chem 1984; 259: 12123-12127.

12. Goldberg MP and Choi DW. Combined oxygen and glucose deprivation in cortical cell culture: calciumdependent and calcium-independent mechanisms of neuronal injury. J Neurosci 1993; 13: 3510-3524.

13. Tauskela JS, Aylsworth A, Hewitt M, et al. Failure and rescue of preconditioning-induced neuroprotection in severe stroke-like insults. Neuropharmacology 2016; 105: 533-542.

14. Brennan AM, Suh SW, Won SJ, et al. NADPH oxidase is the primary source of superoxide induced by NMDA receptor activation. Nat Neurosci 2009; 12: 857-863.

15. Ramnath RR, Strange K and Rosenberg PA. Neuronal injury evoked by depolarizing agents in rat cortical cultures. Neuroscience 1992; 51: 931-939.

16. Hamill OP, Marty A, Neher E, et al. Improved patchclamp techniques for high-resolution current recording from cells and cell-free membrane patches. Pflugers Arch 1981; 391: 85-100.

17. Bourourou M, Heurteaux C and Blondeau N. Alpha-linolenic acid given as enteral or parenteral nutritional intervention against sensorimotor and cognitive deficits in a mouse model of ischemic stroke. Neuropharmacology 2016; 108: 60-72.

18. Fisher M, Feuerstein G, Howells DW, et al. Update of the stroke therapy academic industry roundtable preclinical recommendations. Stroke 2009; 40: 2244-2250.

19. Balkaya M, Krober JM, Rex A, et al. Assessing poststroke behavior in mouse models of focal ischemia. J Cereb Blood Flow Metab 2013; 33: 330-338.

20. Nguemeni C, Delplanque B, Rovere C, et al. Dietary supplementation of alpha-linolenic acid in an enriched rapeseed oil diet protects from stroke. Pharmacol Res 2010; 61: 226-233.

21. Bading H, Ginty DD, Greenberg ME. Regulation of gene expression in hippocampal neurons by distinct calcium signaling pathways. Science 1993; 260: 181-186.

22. Hartley DM, Kurth MC, Bjerkness L, et al. Glutamate receptor-induced $45 \mathrm{Ca} 2+$ accumulation in cortical cell culture correlates with subsequent neuronal degeneration. J Neurosci 1993; 13: 1993-2000.

23. Galluzzi L, Blomgren K and Kroemer G. Mitochondrial membrane permeabilization in neuronal injury. Nat Rev Neurosci 2009; 10: 481-494.

24. Perez-Pinzon MA, Stetler RA and Fiskum G. Novel mitochondrial targets for neuroprotection. J Cereb Blood Flow Metab 2012; 32: 1362-1376.

25. Ehrenberg B, Montana V, Wei MD, et al. Membrane potential can be determined in individual cells from the nernstian distribution of cationic dyes. Biophys J 1988; 53: 785-794.

26. Fiskum G, Murphy AN and Beal MF. Mitochondria in neurodegeneration: acute ischemia and chronic neurodegenerative diseases. J Cereb Blood Flow Metab 1999; 19: 351-369.

27. Lin MT and Beal MF. Mitochondrial dysfunction and oxidative stress in neurodegenerative diseases. Nature 2006; 443: 787-795.

28. Robinson KM, Janes MS and Beckman JS. The selective detection of mitochondrial superoxide by live cell imaging. Nat Protoc 2008; 3: 941-947.

29. Heurteaux C, Bertaina V, Widmann C, et al. $\mathrm{K}^{+}$channel openers prevent global ischemia-induced expression of c-fos, c-jun, heat shock protein, and amyloid b-protein precursor genes and neuronal death in rat hippocampus. Proc Natl Acad Sci U S A 1993; 90: 9431-9435.

30. Szewczyk A and Wojtczak L. Mitochondria as a pharmacological target. Pharmacol Rev 2002; 54: 101-27.

31. Shin WW, Fong WF, Pang SF, et al. Limited blood-brain barrier transport of polyamines. J Neurochem 1985; 44 : 1056-1059.

32. Krueger M, Bechmann I, Immig K, et al. Blood-brain barrier breakdown involves four distinct stages of vascular damage in various models of experimental focal cerebral ischemia. J Cereb Blood Flow Metab 2015; 35: 292-303.

33. Bale G, Mitra S, de Roever I, et al. Oxygen dependency of mitochondrial metabolism indicates outcome of newborn brain injury. J Cereb Blood Flow Metab 2019; 39: 2035-2047.

34. Magistretti PJ and Allaman I. Lactate in the brain: from metabolic end-product to signalling molecule. Nat Rev Neurosci 2018; 19: 235-249.

35. Mazucanti $\mathrm{CH}$, Kawamoto EM, Mattson MP, et al. Activity-dependent neuronal Klotho enhances astrocytic aerobic glycolysis. J Cereb Blood Flow Metab 2019; 39: 1544-1556.

36. Hayakawa K, Esposito E, Wang X, et al. Transfer of mitochondria from astrocytes to neurons after stroke. Nature 2016; 535: 551-555.

37. Borlongan CV, Nguyen H, Lippert T, et al. May the force be with you: transfer of healthy mitochondria from stem cells to stroke cells. J Cereb Blood Flow Metab 2019; 39: 367-370.

38. Rutkai I, Merdzo I, Wunnava SV, et al. Cerebrovascular function and mitochondrial bioenergetics after ischemiareperfusion in male rats. J Cereb Blood Flow Metab 2019; 39: 1056-1068. 
39. Stavrovskaya IG, Narayanan MV, Zhang W, et al. Clinically approved heterocyclics act on a mitochondrial target and reduce stroke-induced pathology. J Exp Med 2004; 200: 211-222.

40. Park MH. The post-translational synthesis of a polyamine-derived amino acid, hypusine, in the eukaryotic translation initiation factor 5A (eIF5A). J Biochem 2006; 139: 161-169.

41. Thomas $\mathrm{T}$ and Thomas TJ. Polyamines in cell growth and cell death: molecular mechanisms and therapeutic applications. Cell Mol Life Sci 2001; 58: 244-258.

42. Saulnier Sholler GL, Gerner EW, Bergendahl G, et al. A phase I trial of DFMO targeting polyamine addiction in patients with relapsed/refractory neuroblastoma. PLoS One 2015; 10: e0127246.

43. Hahm HA, Ettinger DS, Bowling K, et al. Phase I study of $\mathrm{N}(1), \mathrm{N}(11)$-diethylnorspermine in patients with nonsmall cell lung cancer. Clin Cancer Res 2002; 8: 684-690.

44. O'Shaughnessy JA, Demers LM, Jones SE, et al. Alphadifluoromethylornithine as treatment for metastatic breast cancer patients. Clin Cancer Res 1999; 5: 3438-3444.

45. Pepin J, Milord F, Guern C, et al. Difluoromethylornithine for arseno-resistant Trypanosoma brucei gambiense sleeping sickness. Lancet 1987; 2: 1431-1433.

46. Levin VA, Hess KR, Choucair A, et al. Phase III randomized study of postradiotherapy chemotherapy with combination alpha-difluoromethylornithine-PCV versus PCV for anaplastic gliomas. Clin Cancer Res 2003; 9: 981-990.

47. Meyskens FL, Jr. and Gerner EW. Development of difluoromethylornithine (DFMO) as a chemoprevention agent. Clin Cancer Res 1999; 5: 945-951.

48. Tantini B, Fiumana E, Cetrullo S, et al. Involvement of polyamines in apoptosis of cardiac myoblasts in a model of simulated ischemia. J Mol Cell Cardiol 2006; 40: 775-782.

49. Babu GN, Sailor KA, Beck J, et al. Ornithine decarboxylase activity in in vivo and in vitro models of cerebral ischemia. Neurochem Res 2003; 28: 1851-1857.

50. Mathews MB and Hershey JW. The translation factor eIF5A and human cancer. Biochim Biophys Acta 2015; 1849: 836-844.

51. Craig M, Bensinger W, Siegel DSD, et al. Phase 1-2 openlabel, multiple-dose, dose-escalation study to evaluate the safety and tolerability of intravenous infusion of SNS01-
T, a first-in-class modulator of eukaryotic translation initiation factor $5 \mathrm{~A}$ (eIF5A) in patients (pts) with relapsed or refractory B-cell malignancies. J Clin Oncol 2014; 32(15_suppl): TPS8616.

52. Imam S, Mirmira RG and Jaume JC. Eukaryotic translation initiation factor 5A inhibition alters physiopathology and immune responses in a "humanized" transgenic mouse model of type 1 diabetes. Am J Physiol Endocrinol Metab 2014; 306: E791-798.

53. Jasiulionis MG, Luchessi AD, Moreira AG, et al. Inhibition of eukaryotic translation initiation factor $5 \mathrm{~A}$ (eIF5A) hypusination impairs melanoma growth. Cell Biochem Funct 2007; 25: 109-114.

54. Zhao L, Mulligan MK and Nowak TS, Jr. Substrain- and sex-dependent differences in stroke vulnerability in C57BL/6 mice. J Cereb Blood Flow Metab 2019; 39: 426-438.

55. Jakus J, Wolff EC, Park MH, et al. Features of the spermidine-binding site of deoxyhypusine synthase as derived from inhibition studies. Effective inhibition by bis- and mono-guanylated diamines and polyamines. J Biol Chem 1993; 268: 13151-13159.

56. Sommer MN, Bevec D, Klebl B, et al. Screening assay for the identification of deoxyhypusine synthase inhibitors. J Biomol Screen 2004; 9: 434-438.

57. Atkins MB, Redman B, Mier J, et al. A phase I study of CNI-1493, an inhibitor of cytokine release, in combination with high-dose interleukin-2 in patients with renal cancer and melanoma. Clin Cancer Res 2001; 7: 486-492.

58. Hommes D, van den Blink B, Plasse T, et al. Inhibition of stress-activated MAP kinases induces clinical improvement in moderate to severe Crohn's disease. Gastroenterology 2002; 122: 7-14.

59. Dotan I, Rachmilewitz D, Schreiber S, et al. A randomised placebo-controlled multicentre trial of intravenous semapimod $\mathrm{HCl}$ for moderate to severe Crohn's disease. Gut 2010; 59: 760-766.

60. van Westerloo DJ, Rauws EA, Hommes D, et al. PreERCP infusion of semapimod, a mitogen-activated protein kinases inhibitor, lowers post-ERCP hyperamylasemia but not pancreatitis incidence. Gastrointest Endosc 2008; 68: 246-254.

61. Shi L, Rocha M, Leak RK, et al. A new era for stroke therapy: integrating neurovascular protection with optimal reperfusion. J Cereb Blood Flow Metab 2018; 38: 2073-2091. 